\title{
Separating Mechanical and Chemical Contributions to Molecular-Level Friction
}

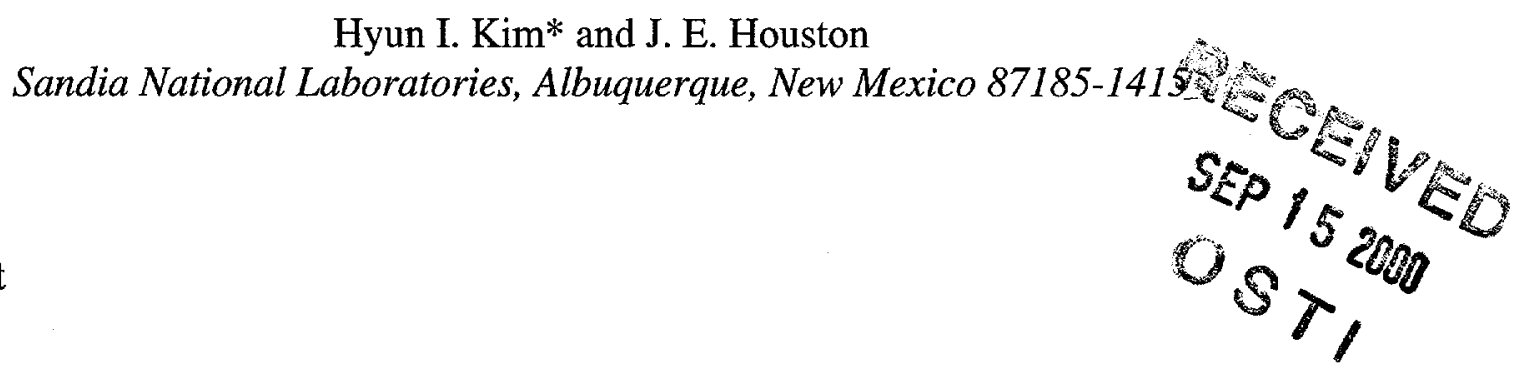

We use force-probe microscopy to study the friction force and the adhesive interaction for molecular monolayers self-assembled on both $\mathrm{Au}$ probe tips and substrate surfaces. By systematically varying the chemical nature of the end groups on these monolayers we have, for the first time, delineated the mechanical and chemical origins of molecular-level friction. We use chemically inert $-\mathrm{CH}_{3}$ groups on both interfacial surfaces to establish the purely mechanical component of the friction and contrast the results with the findings for chemically active $-\mathrm{COOH}$ end-groups. In addition, by using odd or even numbers of methylene groups in the alkyl backbones of the molecules we are able to determine the levels of inter-film and intra-film hydrogen bonding. 


\section{DISCLAIMER}

This report was prepared as an account of work sponsored by an agency of the United States Government. Neither the United States Government nor any agency thereof, nor any of their employees, make any warranty, express or implied, or assumes any legal liability or responsibility for the accuracy, completeness, or usefulness of any information, apparatus, product, or process disclosed, or represents that its use would not infringe privately owned rights. Reference herein to any specific commercial product, process, or service by trade name, trademark, manufacturer, or otherwise does not necessarily constitute or imply its endorsement, recommendation, or favoring by the United States Government or any agency thereof. The views and opinions of authors expressed herein do not necessarily state or reflect those of the United States Government or any agency thereof. 


\section{DISCLAIMER}

Portions of this document may be illegible in electronic image products. Images are produced from the best available original document. 
There has been growing interest recently in the use of self-assembled monolayer (SAM) films as lubricants for such applications as micro-electromechanical machines ${ }^{1}$ and broad discussion of the details of friction at the molecular level. ${ }^{2}$ In this letter we report the use of interfacial force microscopy to study the mechanical and chemical mechanisms that govern friction forces for alkanethiol SAM films grown on Au surfaces. The major focus is on the simultaneous measurement of the adhesive and friction forces for SAM-coated probe and sample surfaces. We then systematically alter the chemical nature of the SAM surfaces and tabulate these forces for various combinations of methyl $\left(-\mathrm{CH}_{3}\right)$ and carboxylic acid $(-\mathrm{COOH})$ end groups. In addition, we alter the end-group orientations by changing the odd-even number of methylene groups in the alkyl backbone. The results allow us to delineate the origins of the friction force starting with a purely mechanical $-\mathrm{CH}_{3}$ vs. $-\mathrm{CH}_{3}$ combination and increasing to the predominantly chemical mechanism involving the making and breaking of inter- and intra-facial bonds. Furthermore, the results indicate that the odd/even-length effect changes the hydrogen bonding between the end groups from predominantly inter-film to strongly intra-film.

For the present studies we use the interfacial force microscope (IFM). This instrument, which has been described in detail elsewhere ${ }^{3}$ is distinguished by its use of a force-feedback, self-balancing sensor. The sensor eliminates the mechanical instability seen in the most often used techniques for studying molecular-level friction and adhesion, i. e., the surface forces apparatus ${ }^{4}$ and the atomic force microscope, ${ }^{5}$ and is able to make quantitative measurements throughout the entire force range including the attractive-force regime. In addition, since the sensor actually balances the torque applied to the tip, normal and lateral forces can be simultaneously obtained by placing a small lateral 
modulation ( $25 \AA$ for the present work) on the tip and separating the force signals in the frequency domain. ${ }^{6}$

The IFM investigation is done in a humidity-controlled environment $(\mathrm{RH} \sim 4 \%)$ on systems consisting of parabolic gold tips (characterized by SEM) and single-crystal gold surfaces that are modified with SAM films with either $-\mathrm{CH}_{3}$ or $-\mathrm{COOH}$ end groups. The odd-COOH film is generated from $1 \mathrm{mM}$ ethanolic solutions of 16mercaptohexadecanoic acid and the even-COOH film from 11-mercaptoundecanoic acid solutions (all solutions use the same ethanolic concentration). The $-\mathrm{CH}_{3}$-terminated films use $n$-hexadecanethiol molecules. The acid-terminated molecules are identical in that both have chemically reactive $-\mathrm{COOH}$ terminal groups exposed at the surface. However, since it is known that the alkyl chains of $n$-alkanethiol films tilt by $\sim 30^{\circ}$ from the surface normal, ${ }^{7}$ the orientation of the last $\mathrm{C}-\mathrm{C}$ unit differs for odd and even films. ${ }^{8}$ Thus, the orientation of the $-\mathrm{COOH}$ terminal groups, and the chemical nature of the surfaces, are different, as illustrated schematically in Fig. 1. Based on this, one can expect that the strength of the hydrogen bonding between films or within a given film will be different. Indeed, IR studies on - $\mathrm{COOH}$-terminated SAMs indicate that the odd films ${ }^{7}$ possess a significant level of intra-film hydrogen bonding compared to the even films. ${ }^{9}$ In addition, we would expect that the intra-film bonding would be obtained at the expense of the interaction between the two films (i.e., inter-film bonding). The overall effect would be that the odd-COOH film would appear to be somewhat passivated by a layer of hydrogen-bonded end groups across the surface. If this model is correct, we would expect a greater interfacial attraction between the even- $\mathrm{COOH}$ pair while the odd$\mathrm{COOH}$ combination would be weaker. We will use data of the variation of the lateral 
friction force vs. the interfacial adhesion force to delineate the mechanical from the chemical nature of the resulting molecular friction and determine the degree to which the model of Fig. 1 is correct. According to this model, we would also expect the contact potential difference (CPD) to be different for odd- and even-COOH films since CPD is directly proportional to electric dipole magnitude normal to the surface. CPD is measured by applying a triangular-sweep voltage between the sample and probe and then determining the applied potential necessary to null the electric field and the interfacial force between the two surfaces. ${ }^{10}$

We show in Fig. 2 a compilation of friction vs. adhesive forces for the various end-group combinations outlined above. Here, negative force values indicate an attractive interfacial interaction while positive values represent repulsion. The peak negative adhesive-force values for each curve can be used to estimate the work of adhesion for the various end-group combinations. However, in this brief report we will use these force values only as a qualitative measure of the level of the interfacial interaction and to help evaluate the mechanical vs. chemical aspects of the molecularlevel friction.

From the individual friction vs. adhesive force curves shown in Fig. 2, we find that the maximum adhesive forces are approximately the same for all combinations except the even- $\mathrm{COOH}$ pair, indicating that these pairs interact by predominantly van der Waals (VdW) forces. However, the even- $\mathrm{COOH}$ pair shows a considerable increase in interfacial bonding. This bonding involves hydrogen (acid/base) bonds formed between the interacting $-\mathrm{COOH}$ groups terminating the two interfacial films. Each $-\mathrm{COOH}$ group represents a reasonably strong acid at the $-\mathrm{OH}$ site and a weak base due to the lone pair at 
the $-\mathrm{OH}$ site. From these results, we immediately see that the interfacial or inter-film bonding is dramatically reduced for the odd- $\mathrm{COOH}$ pair. The odd/even effect for the $-\mathrm{COOH}$ interfacial bonding is corroborated by an additional piece of information; The measured contact potential difference (CPD) is $640 \mathrm{mV}$ for the even-COOH film, which is significantly greater than the $150 \mathrm{mV}$ measured for odd-COOH. The even-COOH value is in good agreement with that previously measured $(533 \mathrm{mV})^{10}$ and with the value calculated $(570 \mathrm{mV})$ using the normal component of the group electric dipole moment. In analogy to the water molecule itself, the dipole moment for the - $\mathrm{COOH}$ end group is dominated by the presence of oxygen lone pairs. As a result, and from the schematic model of Fig. 1, it is clear that the even-COOH film will have a higher $\mathrm{CPD}$ value, which is our finding.

We now look at the frictional properties in order to separate the mechanical and chemical processes. The first thing to note from Fig. 2 is the nonlinear behavior of the curves. In all cases, the friction begins near the bottom of the adhesive excursion as the interfacial-interaction force just begins to decrease toward repulsion. The friction force rises rapidly in this region and appears to approach a constant slope as the interfacialinteraction force turns repulsive, leading to a constant friction coefficient. Earlier work on SAM films at higher repulsive forces also observed a linear relationship. ${ }^{11}$ The nonlinear behavior, especially for the even- $\mathrm{COOH}$ pair is very reminiscent of the Johnson, Kendall, Roberts (JKR) model for the variation in contact-area for the interaction of a spherical probe and elastic substrate with contact-adhesion forces. ${ }^{12}$ In fact, this behavior has been observed for AFM friction measurements on mica. ${ }^{13}$ The 
friction-load plot for the $\mathrm{CH}_{3}-\mathrm{CH}_{3}$ interface serves as a reference for the purely mechanical portion of the friction for the other combinations of Fig. 2.

For the $\mathrm{CH}_{3}-\mathrm{COOH}$ interactions, the even pair exhibits frictional forces that are only slightly greater than those for the $\mathrm{CH}_{3}-\mathrm{CH}_{3}$ pair, indicating that its origin is predominantly mechanical. This implies that, as expected, there is no inter-film chemical interaction and the intra-film hydrogen bonding is at best weak. In contrast, the odd - $\mathrm{CH}_{3}$ $-\mathrm{COOH}$ pair shows a significant increase in friction while the level of adhesion stays near that for the $\mathrm{CH}_{3}-\mathrm{CH}_{3}$ pair. As was suggested by the model of Fig. 1, this result supports the earlier finding ${ }^{7,9}$ that the odd-COOH film possesses a significant level of intra-film hydrogen bonding, which would be perturbed under sliding contact. The making and breaking of these bonds during sliding will provide an additional energy-loss mechanism and lead to higher friction.

For the $\mathrm{COOH}-\mathrm{COOH}$ interactions, the odd pair exhibits much higher frictional forces than the odd $\mathrm{CH}_{3}-\mathrm{COOH}$ pair, since one more layer of - $\mathrm{COOH}$ groups is present at the sliding interface, although the adhesion stays virtually identical. In keeping with this doubling of the bonded layers, the magnitude of the friction force is also very nearly doubled. In contrast, the even- $\mathrm{COOH}$ pair shows a similar level of friction but a considerably increased level of interfacial adhesion. As discussed earlier, this strong inter-film chemical interaction results in a similar level of friction in the repulsive regime to that of the odd $-\mathrm{COOH}$ pair. In each case the bonding involves both films. However, the rate of the friction increase is greater for the odd pair and its friction-load plot crosses that of the even pair at around $0.6 \mu \mathrm{N}$. The clear implication is that the total energy involved in the intra-film bonding for the odd - $\mathrm{COOH}$ pair is somewhat larger than that 
of the even $-\mathrm{COOH}$ pair. This could either be the result of stronger hydrogen bonding per molecular pair or to a greater density of bonds across the film surface.

In conclusion, we have used force-probe microscopy to study the friction and adhesive interactions for molecular monolayers self-assembled on both a Au probe tip and a substrate surface. By systematically varying the chemical nature of the end groups on these monolayers we have, for the first time, delineated the mechanical and chemical origins of molecular-level friction. The mechanical component is established from the results of the chemically inert $-\mathrm{CH}_{3}$ pair and contrasted with the findings for chemically active $-\mathrm{COOH}$ end-group combinations. By taking advantage of the odd/even effect on $-\mathrm{COOH}$ group orientation we are also able to obtain valuable information on the relative levels of inter- and intra-film hydrogen bonding. We find that the even- $\mathrm{COOH}$ films expose a combination of hydrogen sites and the oxygen lone pair and make inter-film hydrogen bonding possible, while the orientation of the odd-COOH combination facilitates intra-film bonding. Simultaneous friction and adhesive-force measurements such as those presented here will significantly expand our ability to analyze the details of molecular-friction and aid in achieving the ability to tailor the chemistry of interfacial systems for specific tribological applications.

\section{Acknowledgment}

We thank James Kushmerick, Dale Huber and Bruce Bunker for valuable discussions. This work was supported by the US Department of Energy under Contract DE-AC0494AL85000. Sandia is a multiprogram laboratory operated by Sandia Corporation, a Lockheed-Martin Company, for the US Department of Energy. 


\section{References}

(1) Maboudian, R. Surf. Sci. Rep. 1998, 30, 207.

(2) Persson, B. N. J. Sliding Friction; Springer: Berlin, 1998.

(3) Joyce, S. A.; Houston, J. E. Rev. Sci. Instrum. 1991, 62, 710-715.

(4) Israelachvili, J. Intermolecular \& Surface Forces; Academic Press: New York, 1992.

(5) Binnig, G.; Quate, C. F.; Gerber, C. Phys. Rev. Lett. 1986, 56, 930.

(6) Kim, H. I.; Boiadjiev, V.; Houston, J. E.; Zhu, X.-Y.; Kiely, J. D. Tribol. Lett., submitted.

(7) Nuzzo, R. G.; Dubois, L. H.; Allara, D. L. J. Am Chem. Soc. 1990, 112, 558.

(8) Wong, S. S.; Takano, H.; Porter, M. D. Anal. Chem. 1998, 70, 5209.

(9) Sun, L.; Kepley, L. J.; Crooks, R. M. Langmuir 1992, 8, 2101.

(10) Thomas, R. C.; Tangyungyong, P.; Houston, J. E.; Michalske, T. A.; Crooks, R. M. J. Phys. Chem. 1994, 98, 4493.

(11) Kiely, J. D.; Houston, J. E. Langmuir 1999, 15, 4513.

(12) Johnson, K. L.; Kendall, K.; Roberts, A. D. Proc. Roy. Soc. Lond. A 1971, 324, 301.

(13) Carpick, R. W.; Agrait, N.; Ogletree, D. F.; Salmeron, M. J. Vac. Sci. Technol. B $1996,14,1289$. 


\section{Figure Captions}

Figure 1. A schematic representation of the $-\mathrm{COOH}$ end-group orientations for alkanethiol SAMs having both odd and even numbers of methylene groups.

Figure 2. Plots of the lateral friction force vs. interfacial force for various end-group combinations. 

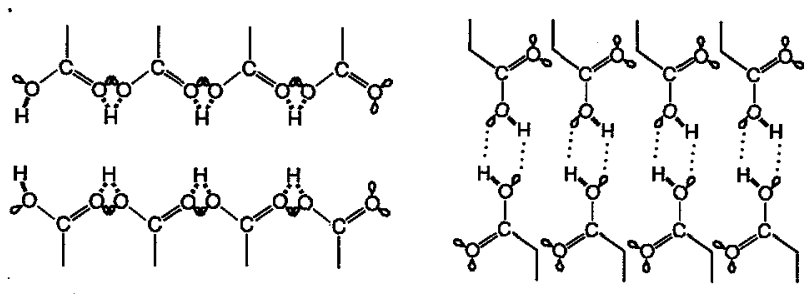

odd- $\mathrm{COOH}$ even- $\mathrm{COOH}$ odd-COOH even-COOH

Figure 1, Kim and Houston 


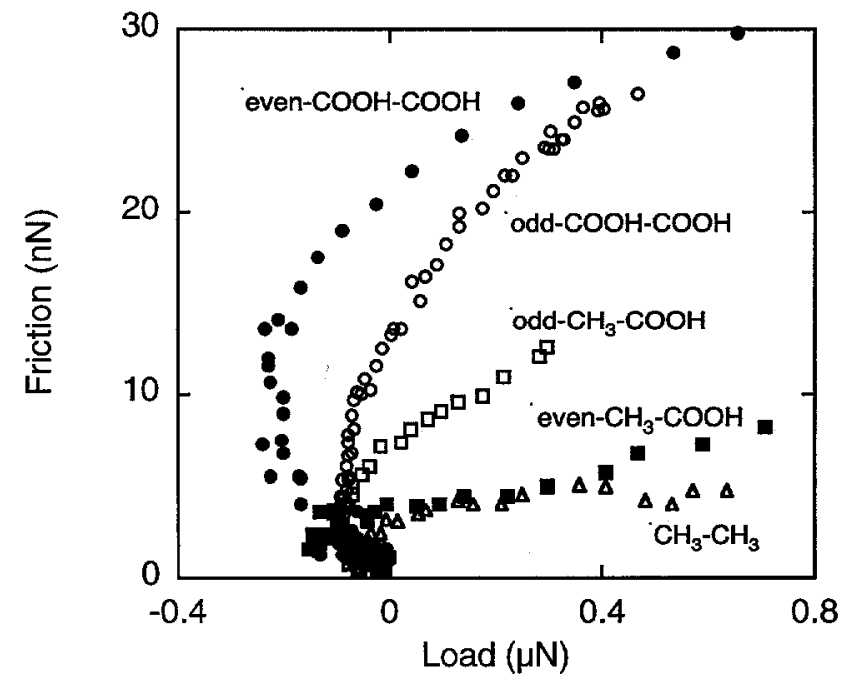

Figure 2, Kim and Houston 